\title{
Schelling y el nacimiento de la conciencia trágica moderna ${ }^{1}$
}

\author{
Crescenciano Grave \\ Universidad Nacional Autónoma de México \\ kenxano@hotmail.com
}

En Schelling - desde y frente a la tradición moderna inaugurada por Descartes y fundamentada por Kant - la filosofía muta el modo de afirmarse a sí misma. Esta mutación afirmativa es la que - proponemos - constituye el nacimiento de la conciencia trágica en la filosofía moderna. El nacimiento se consigue por la alteración del sentido del sujeto; el sujeto, antes que subjetividad consciente, es la actividad general e infinita que queriéndose a sí misma se objetiva dando lugar a la totalidad de los entes. El sujeto es el Ser que, sin presentarse nunca como tal, actúa en toda presencia y al cual nunca podemos definir sino tan sólo referir desde el despliegue de un pensamiento preñado y atravesado por la contradicción. Pretendemos mostrar esto en tres puntos: el principio trágico del ser, la experiencia trágica de la historia y las manifestaciones del ser.

\section{El principio trágico del ser}

¿A qué se refiere Schelling con el sujeto general e infinito? El sujeto que aquí se piensa señaladamente es el Ser como condición de posi-

\footnotetext{
${ }^{1}$ Este ensayo forma parte de una investigación más amplia sobre la relación entre la historia del pensamiento moderno y la constitución del sistema filosófico en Schelling y Hegel. Esta relación se explora principalmente siguiendo las líneas que cada uno de estos autores exploran en sus respectivas historias del pensamiento. Las Lecciones sobre la historia de la filosofia de Hegel y las Lecciones muniquesas para la historia de la filosofia moderna de Schelling. En esta obra póstuma Schelling se confronta con las primeras versiones sistemáticas de su propia filosofía, esto es, el idealismo trascendental y la filosofía de la naturaleza. Nuestras reflexiones sobre este volver de Schelling a sí mismo nos permiten sostener nuestra interpretación de que con él nace la conciencia trágica en la filosofía moderna.
} 
bilidad de que todo se presente. Es el Ser que no pudiendo presentarse nunca en su unidad como tal, se presenta siempre alterándose en la diversidad de los entes. El sujeto es, en términos de Schelling, el Ser como nada queriendo ser todo.

El sujeto en cuanto que es pensado en su pura sustancialidad está todavía libre de todo ser y, aunque no sea una nada, sin embargo es como nada. No es una nada porque es sujeto; es como nada, porque no es objeto y no existe en el ser objetivo. Sin embargo, el sujeto no puede permanecer en esta abstracción; para él es, por decirlo así, natural quererse a sí mismo como algo, y como objeto (1993; 197).

La conciencia trágica nace llevando al pensamiento y al lenguaje filosóficos a los límites con lo inefable. Se trata de pensar, nombrándolo como sujeto, al inicio de todo ser objetivo. El inicio es como nada porque precisamente no es nada objetivo, no es ni esto ni aquello; pero no es una nada porque en este sujeto general e infinito se activa naturalmente la voluntad, el quererse a sí mismo. Él no puede obtener lo que quiere - a sí mismo - si permanece como nada, por lo tanto, por su propio querer el sujeto llega a ser objetivándose como algo, como esto y aquello. La vecindad de este pensamiento con lo inefable radica en que hay que pensar a este sujeto como nada definido y, al mismo tiempo, queriendo-se ser objetivamente todo. El sujeto es la voluntad escindida en sí misma: ella es lo que quiere y lo querido de tal modo que el principio de toda presencia y la presencia misma está signada por la contradicción entre la libertad que, como actividad productiva, quiere, y la necesidad en la que, como producción efectiva, se quiere y se realiza.

La conversión voluntaria del sujeto en objeto no lo fija definitivamente, sino que el sujeto, por su voluntad infinita, se objetiva finitamente, y esta realización, precisamente por ser finita, no lo satisface, por lo tanto, se ve impulsado por sí mismo a sobrepasar esta objetivación en otra y así, in-finitamente.

El pensamiento sobre el sujeto se concentra en una «autoposición infinita» no sólo en sentido negativo sino, sobre todo, en sentido positivo. La autoposición infinita del sujeto en sentido negativo significa que él no es finito, no está determinado; y en sentido positivo significa 
que el sujeto tiene el poder de hacerse finito, de autodeterminarse, y esta finitud lo determina objetivamente, pero no lo fija definitivamente en tanto, por sí mismo: «cada vez que se hace finito u objeto se eleva de nuevo a una potencia más alta de la subjetividad» (1993; 197).

La conversión del sujeto en objeto no anula su impulso de quererse; el sujeto como voluntad de ser no se colma y, en y desde sus propias objetivaciones, él continúa transformándose queriendo ser más en un devenir indetenible y progresivo en el que el sujeto va potenciando cada vez más su voluntad in-finita de ser.

Desde este pensamiento en donde el sujeto abandona la prisión trascendental yéndose al fondo para, como Ser, fundamentar en su voluntad su propio devenir en los entes objetivos, Schelling alza su sistema como filosofía de la naturaleza. Y este alzado requiere de un principio que lo sustente. El principio es aquello que sostiene al sistema y éste se estructura como el despliegue de aquél.

El comienzo es naturalmente el primer hacerse algo del sujeto, el primer objetivarse; a éste seguía la infinitud del sujeto, según la cual a cada objetivarse no sigue inmediatamente más que una potencia superior de la subjetividad - con la primera objetivación se ponía el fundamento de toda elevación posterior y, consiguientemente, del movimiento mismo $(1993 ; 197)$.

Al objetivarse el sujeto no se desvanece la subjetividad sino que ésta permanece persistiendo como actividad superadora de sí mismo en tanto toda objetivación es una realización limitada del querer del propio sujeto. Y con la primera realización de este querer - la materia como potencia de formación - se pone la base de toda evolución posterior del sujeto a través de sucesivas objetivaciones con lo cual se da inicio al movimiento por el que el Ser deviene queriéndose a sí mismo en la diversidad de los entes.

Y este devenir por el que el Ser llega a la objetividad de sí mismo en los entes está posibilitado ontológicamente por la contradicción. El ser aún no objetivo pensado en su pura esencialidad es como nada: este ser como nada expresa la negación de todo ser objetivo porque nada de lo que se presenta desde su propia actividad lo realiza consumándolo. Pero, al ser como nada, el Sujeto no es nada sino 
que, también, es como algo: un ser que se le añade, advenedizo, contingente. El ser como algo del Sujeto indica un ser atraído. El sujeto sujetado al ser como nada se ve atraído por su misma voluntad a ser como algo: el objeto se presenta por un movimiento inmanente de la esencia para añadirse la contingencia.

El sujeto, en su pura naturalidad o esencialidad, es como nada, no posee ninguna característica distintiva, no es - dice Schelling«más que Él mismo y, por tanto, plena libertad respecto de todo ser y frente a todo ser». Pero a este ser o esencia libre de toda objetivación «le es inevitable atraerse a sí mismo», atracción por la cual el sujeto se convierte en objeto saliendo por su propia esencia de su misma esencia, esto es, existiendo.

El sujeto, al objetivarse existiendo, ya no es como nada ni como algo sino que se determina como objeto. Y en la autoatracción del sujeto a la existencia, yace - dice Schelling - el origen del ser objetivo en general $(1993 ; 198)$.

Con esto aparece el primer rasgo trágico de la filosofía de Schelling: el sujeto sólo puede ser para sí a través de su objetivación, es decir, mediante el dejar atrás su esencia, por lo tanto, el sujeto nunca se posee a sí mismo tal y como es en su esencia sino que accede a sí mismo siempre desde su contingencia como existente. «El sujeto nunca puede llegar a poseerse como aquello que es, ya que justamente al atraerse a sí mismo se convierte en otro» $(1993 ; 198)$. Al buscar recuperarse en su identidad se encuentra en su alteridad.

El pliegue de voluntad que es el Ser como nada se objetiva desplegándose como algo y, al replegarse para sí, hay un resto que no retorna porque el sujeto se refleja como algo y nunca como nada, que es lo que esencialmente es. En sus objetivaciones el ser se escapa de sí existiendo y desde la existencia nunca puede retornar plenamente a sí mismo.

Esta es la contradicción fundamental, podemos decir que es la desdicha de todo ser - pues o bien él se abandona, y entonces es como nada, o bien se atrae a sí mismo, y entonces es otro y distinto de sí mismo; ya no está como antes en una relación de naturalidad con el ser, sino que se turba ante él, y él mismo experimenta este ser como un ser añadido y, por tanto, contingente (1993; 198). 
El abandonarse del sujeto a su ser como nada implicaría la supresión de la existencia objetiva y, entonces sí, nada sería. Y al atraerse a sí convirtiéndose en ser objetivo el Ser se altera y se turba a sí mismo al añadir lo contingente a su esencia. En el principio el Sujeto o Ser esencial se juega en la encrucijada de la nada y la contingencia.

El segundo rasgo trágico que Schelling introduce en la filosofía es el siguiente: puesto que todo lo que es, es resultado del desalojo que la esencia atrae sobre sí existiendo, entonces ella, para no abandonarse en la nada, tiene necesariamente que convertirse en lo otro de sí, y en la medida en que lo que ella es para sí es sólo reflejándose desde lo otro, entonces todo su ser objetivo es contingente. Esto es lo que Schelling llama la desdicha de todo ser.

Todo lo que es objetivamente presente es resultado del atraerse queriéndose a sí del sujeto esencial que, por lo mismo, se altera deviniendo diferente, de tal modo que ya nunca es lo que esencialmente era sino que, abandonando la eternidad sumergiéndose en el tiempo, es siempre la continua turbación contingente de sí mismo en la existencia.

Al pensar a lo existente como alteración contingente de la esencia, se patentiza que el lenguaje de la metafísica trágica tiene una de sus orillas en la inefabilidad y la otra en la plenitud de significado: la esencia que en tanto tal se sustrae a todo pensamiento determinado termina convirtiéndose en la afirmación contingente de todo, afirmación que se desplegará hasta formarse como vida que, atravesando a la totalidad, se levanta conscientemente en la grandiosa precariedad de la existencia humana.

$\mathrm{Al}$ abandonar a la esencia para existir propiamente, el origen del ente se vuelve contingente. Este origen es la contingencia original o disonancia porque la esencia se altera a sí misma en la existencia: el sujeto libre de todo ser, para ser esto y esto otro tiene necesariamente que convertirse en objeto. La esencia como lo mismo y libre, se altera deviniendo necesariamente diferente respecto de sí misma. Esta disonancia se manifiesta también como la contraposición de lo infinito con lo finito. El sujeto como infinitud o libre de todo ser objetivo, se pone como lo otro de sí o-poniéndose en lo finito. En la mera infinitud se diluye todo ser por lo cual el sujeto infinito sólo puede ponerse limitándose u oponiéndose como finito, esto es, deviniendo ser objetivo. 
¿Cómo se perfila desde aquí lo que podemos llamar la tragedia del ser? El sujeto, en tanto Ser, es la esencia infinita y libre que, queriéndose a sí mismo, necesariamente deviene objeto o ente contingente y finito. El sujeto como tal, dice Schelling, «no está presente a sí mismo» (1993; 199) y, en cuanto se quiere poseer, se convierte en objeto: la esencia subjetiva se o-pone a sí misma como contingencia objetiva queriendo ser para sí misma. Al convertirse en contingencia objetiva, la esencia infinita del sujeto no se supera ya que lo objetivo es para el sujeto cuando éste retorna a su propia esencia; cuando se pone como esencia frente a lo objetivo contingente que él mismo no era antes. Pero en la medida en que el sujeto sólo puede ser para sí a través del objeto en el que él mismo se ha convertido, el retorno del sujeto a sí mismo en la total transparencia de su esencia nunca se consuma del todo. Con la subjetividad buscando regresar a sí se potencia infinitamente a través de sus propias objetivaciones finitas.

El sujeto se derrama en lo objetivo pero este proceso no cancela su esencia que, en tanto infinita, se pone por sí y se repone para sí a través de su propia existencia contingente. Esta potenciación del sujeto se sostiene en lo que Schelling denomina «acto fatal de autoatraerse» el Ser o sujeto esencial que no puede «contenerse a sí mismo», dando lugar a que haya vida. Y a través de la vida originariamente contingente, la subjetividad esencial existe potenciándose, buscando llegar a sí misma como esencia.

Lo que activa la tragedia del Ser es la bifurcación entre la quietud (y permanecer como nada) y el quererse a sí mismo y alterarse, devenir otro de sí mismo; lo trágico es la contradicción originaria que aparece en tanto la mismidad esencial, para afirmarse, tiene que diferenciarse existiendo en la diversidad contingente. Esta contradicción es la que se potencia deviniendo vida. La vida en general es la presencia existente de la esencia que dura en lo transitorio renovándose eternamente y, por lo tanto, nunca posicionándose en sí como lo que en sí misma es.

El Ser se quiere, al quererse se atrae objetivándose, al objetivarse se altera y por su necesaria alteración el ser nunca se ob-tiene tal y como se quiere. Pero al alterarse el ser abandona su quietud como nada deviniendo vida: la vida, como resultado del querer y del poder del sujeto de existir como otro, es la contingencia en la que el Ser se redime temporalmente de su ser como nada. 
El limitarse - o ser cautivo de sí mismo - en lo finito y, a la vez, permanecer como sujeto - seguir queriendo ser in-finitamente en sus objetivaciones - significa que el ser como nada de la esencia permanece acechante en todo ser algo de la misma. Es decir, en la medida en que la esencia - su ser como nada - del sujeto no se desvanece en ninguna de sus objetivaciones reales sino que permanece potenciándose en ellas, este ser como nada pende como una amenaza incesante sobre todo lo existente.

Esta es la tensión trágica que se descubre en la metafísica de Schelling y con la cual nace la conciencia trágica en la filosofía moderna: en el devenir de todo lo existente se potencia sucesivamente el quererse de la esencia y en su mayor complejidad en el alcance de sus objetivaciones, es decir, en las que mejor la expresan menor es también la duración de sus individuos. En los organismos vivos individuales se alcanza una mayor perfección en la manifestación de la esencia pero también una mayor intensidad de la finitud; y en los individuos vivos conscientes esta intensidad se abisma en la angustia consciente de su ser como nada que es al mismo tiempo la potencia que lo impulsa a la posesión de sí mismo como libertad.

\section{La experiencia trágica de la historia}

El pensamiento sobre el sujeto, como principio contradictorio de todo, no se pierde en la vana especulación en la medida en que alumbra la experiencia del hombre en la historia. El sujeto se reposiciona consciente de sí mismo en el ser humano. La consumación de la naturaleza en el ser que devendrá humano tiene como resultado la finitud consciente de sí misma del sujeto. La subjetividad infinitamente libre que da lugar a las organizaciones originales de la naturaleza, se presenta ahora existiendo bajo la forma de la finitud libre de la esencia humana. Lo mismo que se realiza deviniendo naturaleza concluida con la aparición del hombre es lo que, desde la apropiación y reproducción consciente de la subjetividad, se repite diferentemente en el ámbito ideal - sabiendo de sí- en la historia. La repetición diferente del devenir del ser en la historia es el comienzo y despliegue del mundo espiritual desde la libertad finita de la exis- 
tencia humana. Todo lo que llega a ser desde la existencia humana libre tiene la marca de la finitud que, desplegándose en el mundo histórico, alzará a éste signado por la oposición de la libertad y la necesidad.

El sujeto, al que hemos definido como elevándose por encima de toda la naturaleza, es inmediatamente sólo saber puro, en cuanto tal infinito y en completa libertad. En esa medida está de nuevo en el mismo punto en que estaba el primer sujeto puesto en su pura libertad e infinitud, pero él está en una relación inmediata con un ser finito y limitado, con la esencia humana, y puesto que no puede menos de llegar a ser su alma inmediata, se ve forzado también a participar en todas sus determinaciones, relaciones y limitaciones. De esta manera, al entrar en todas las formas de la finitud, se ve obligado a hacerse finito a sí mismo, y aunque él mismo permanece siempre ideal, sin embargo, se ve forzado a enredarse en la necesidad (ideal) que domina en el ámbito del ser o de lo real $(1993 ; 209)$.

La primera potencia que en y desde el mundo histórico se fundamenta es la experiencia - filosofía teórica - o sistema de las representaciones necesarias y de los conceptos que «determina el mundo objetivo para la conciencia humana» $(1993 ; 209)$. El saber, en sí libre e infinito, se une a lo finito vinculándose al mundo real y con este vínculo «se confunde con la necesidad y aparece entonces él mismo como un saber necesario y atado» $(1993 ; 210)$. En la representación cognoscitiva el sujeto consciente es la condición de posibilidad que, para dar cuenta verdaderamente de lo que es, tiene necesariamente que atarse a la presencia objetiva de los fenómenos. Desde esta atadura a la presencia para determinarla representándosela, el sujeto, como ser humano, retorna a sí como libre y se eleva a su segunda potencia colocándose fuera de la necesidad y, por lo tanto, dominándola y comprendiéndola.

La oposición que recorría toda la serie recibe aquí su más alta expresión como oposición de necesidad y libertad. La necesidad es aquello con lo que el hombre tiene que ver en su conocer y a lo que él está sometido en su conocer. La libertad es libertad de hacer y de obrar. Todo obrar 
presupone un conocer, o en el obrar vuelve a hacer objetivo el hombre su propio conocer y se eleva por encima del mismo $(1993 ; 210)$.

El actuar, como objetivación del saber, es el medio por el cual el Sujeto o Ser primordial se vuelca convirtiéndose en mundo objetivo humano e histórico. En el tránsito del saber al obrar se consigue objetivar la necesidad al mismo tiempo que el sujeto se libera de esta necesidad constituyéndose en libre para actuar. La oposición originaria no se cancela sino que se pone como oposición mundana de libertad y necesidad cuya fertilidad se extiende tejiendo el tramado histórico en el que actúa la especie humana.

Se transita así de la filosofía teórica a la filosofía práctica. Aquí el Sujeto ya no se objetiva originariamente en la naturaleza sino que, escindido de las objetivaciones de ésta potenciando su subjetividad, se realiza en la historia desde la libertad del hombre. Y la manifestación de esta realización es la libertad moral del hombre, la oposición entre el bien y el mal, el sentido de esta oposición y el Estado como producto y mediación subordinado de la lucha que la humanidad establece entre libertad y necesidad. La filosofía práctica tiene su esfera propia en la historia concebida como el proceso en el que el Ser implica a toda la humanidad alzando una realidad en la que él mismo aparece en su máxima potenciación subjetiva.

La potenciación de la actividad del sujeto que propicia el salto de la naturaleza a la historia implica la transformación de la filosofía que pasa de ser Filosofía de la naturaleza a ser Filosofía de la historia.

Schelling construye una peculiar visión trágica de la historia en donde la libertad se sitúa en la acción del hombre y la necesidad en las consecuencias que esta acción tiene no sólo para el agente sino para el grupo e, inclusive, para la humanidad en su conjunto. El hombre es el agente de la historia, pero ésta, como el tramado de acciones y consecuencias o como el tejido de la oposición entre libertad y necesidad, se escapa del control o dominio de cualquier individuo o grupo determinado asumiendo su propia legalidad.

Aquí [en la historia] donde se pone de relieve la más alta y la más trágica disonancia, en la que el abuso de la libertad nos enseña a apelar de nuevo a la necesidad, el hombre se ve forzado a reconocer algo que 
es superior a la libertad humana. El deber mismo no le puede exigir al hombre, tan pronto como ha tomado una decisión, que esté tranquilo sobre las consecuencias de su acción, si no pudiese ser consciente de que su acción, ciertamente, depende de él, de su libertad; pero las consecuencias, o aquello que se desarrolla a partir de esta acción para toda su especie, dependen de algo distinto y más elevado que mantiene y afirma una legalidad más alta a través del modo de acción más libre y anárquico del individuo $(1993 ; 211)$.

Con la idea del vínculo trágico entre la libertad del individuo en su acción y la necesidad de las consecuencias, Schelling pretende dejar de lado «una concepción desconsoladora y desesperada de la historia» al mismo tiempo que perfila una peculiar relación causaefecto en el ámbito de la misma: la libertad del hombre es la causa de la acción pero los efectos de ésta no están determinados por el agente del caso sino que se enredan en una trama superior llamada, por Schelling, providencia, en el sentido de disposición de los acontecimientos que tejen un mundo en el cual y frente al cual nos decidimos o intervenimos trocando nuestra libertad en necesidad, en destino. Esta subsunción o incorporación de las consecuencias de los actos en una legalidad más alta constituye la revelación del Ser en el transcurso de la historia, revelación que, impulsada por el hombre, no está, sin embargo, sujeta a su dominio. En el tramado histórico se anuda la libertad con la necesidad porque, frente al conflicto de la libertad humana concreta con la necesidad objetiva, el Sujeto que trama la necesidad de la historia «no se objetiva realmente bajo ninguna forma sino que permanece sujeto puro, libre, indiferente $y$, por tanto, verdaderamente infinito» $(1993 ; 211)$. En la historia acontecida desde el obrar de los hombres cuyas consecuencias, no obstante la libertad individual, se escapan del dominio de cada uno tejiendo una necesidad conflictiva con la libertad humana, se revela el Sujeto primordial y, por lo tanto, exento de una objetivación que lo consume o realice completamente. En la historia el Ser se manifiesta como «lo que ya era al principio» $(1993 ; 212)$ : espíritu que, al realizarse, se muestra como tal y, por lo tanto, irreducible a cualquier objetivación.

La historia no puede consumarse: los distintos mundos surten 
a los hombres que les ha tocado en suerte vivirlos, de preocupaciones, desdichas, injusticias, pero también de motivos para confrontarse con la necesidad de su época afirmando su libertad en la lucha contra lo aciago de su época para lograr introducir entre sus resquicios algunos momentos de alegría.

\section{Las manifestaciones del Ser}

El Ser como Sujeto tanto de la naturaleza como de la historia no se identifica con ninguna de sus objetivaciones y puesto que sólo lo objetivo puede ser representado por la conciencia, el Ser como tal nunca es aprehendido plenamente por ésta. Esto no quiere decir que el Sujeto permanezca encerrado en una oscuridad impenetrable; el Ser - dice Schelling retomando una expresión de Leibniz - fulgura o se manifiesta; el Ser se revela. El Ser se relaciona con la conciencia no determinado en representaciones objetivas sino mediante sus manifestaciones que ocurren tomando como instrumento o medio a la conciencia misma:

[...] tales manifestaciones o [...] tales fulguraciones de aquello que es lo más alto, de lo sublime por encima de todo, son comprobables en la conciencia humana como manifestaciones en las que el yo humano se comporta como instrumento u órgano de aquello que es lo más alto; pues lo que meramente se manifiesta no actúa inmediatamente, sino sólo por medio de otro $(1993 ; 212)$.

El sujeto infinito, para manifestarse relacionándose con la conciencia, toma a esta misma como medio para fulgurar desde y en el arte, la religión y la filosofía. Estas tres son las creaciones en las cuales lo que se manifiesta - lo sublime por encima de todo- aparece como la misma unidad que deviene diferente en la naturaleza y en la historia.

La unidad del Sujeto es la mismidad que diferenciándose es la causa última de la naturaleza o mundo real, y principio rector sobre la historia o mundo ideal - realización del sujeto en el hombre en la que lo que éste objetiva es para sí. El sujeto media entre ambos mundos a la vez que los comprende como uno. En cuanto causa 
productora de todo, el Sujeto se manifiesta en el hombre como producción artística. La creación artística se muestra, primero, como arte plástico en el cual se reproduce el poder del Sujeto sobre la materia al transformarla haciéndola expresión del espíritu o de las ideas más elevadas, y, segundo, como arte poético; en éste el sujeto se manifiesta propiamente como espíritu en el que reside el poder de crear la materia misma como expresión de las ideas, esto es, el lenguaje. En tanto poder de transformar la materia para que éste dé forma, expresándolas, a las ideas, la verdad del arte plástico no se localiza en la conformidad con el modelo sino en la producción misma por la cual las obras plásticas parecen haber sido creadas por la misma naturaleza. El arte plástico manifiesta al creador de la misma naturaleza $(1993 ; 212)$. Este creador se subjetiva conscientemente en la historia y en el lenguaje, y el conflicto surgido de la afirmación de la libertad individual y la incorporación de sus consecuencias en una trama necesaria, es lo que se acoge en las formas de la poesía, concretamente en la tragedia.

En la obra suprema, que es la poesía unida al arte, en la obra suprema del arte poético - la tragedia - aparece el espíritu del poeta en medio del ímpetu de pasiones que luchan ciegamente entre sí, en donde para los actores mismos enmudece la voz de la razón y en donde la arbitrariedad y la anarquía arraigándose cada vez más se transforman finalmente en una necesidad implacable; en medio de todos estos movimientos aparece, repito, el espíritu del poeta como la luz tenue que brilla todavía solo, como el sujeto que permanece arriba solo, inmóvil incluso dentro del movimiento más violento, como una sabia providencia que, finalmente, es capaz de conducir hacia una salida satisfactoria a lo que está al máximo de contradicciones (1993; 213).

En la creación de la poesía trágica acontece una manifestación simbólica del conflicto metafísico del principio que siendo como nada quiere serlo todo, conflicto que, a su vez, se reproduce distinguiéndose históricamente como lucha o colisión entre la libertad y la necesidad, y ante esta manifestación la razón estalla en lo inefable y, no obstante, el poeta - como el Sujeto mismo - permanece sereno ante la violencia de las pasiones propiciando, al llevar a la contra- 
dicción a manifestarse como tal, asentir a ella. Manifestando lo más alto - el destino forjado desde la afirmación de la propia libertadla poesía acontece como tragedia y ésta se manifiesta ante y en la conciencia como el genio propio del arte.

El arte - ya sea creación transformadora de la materia para expresar ideas o productora de las ideas bajo una forma lingüísticaes el lado objetivo de la actividad humana. Frente a él, como el lado subjetivo de esta actividad, se levanta la religión que, dice Schelling, no pone un ser sino que abre la conciencia a la consideración de los entes como no-ser en relación al Ser supremo. En la religión se representa la finitud de los entes como nada si los vemos desde su sometimiento al Ser infinito por el cual ellos aparecen siendo. «Por tanto, aquí se manifiesta ese sujeto supremo como aquel frente al cual todo se hunde en la nada, y como tal él se manifiesta en el entusiasmo de aquellos héroes ético-religiosos, a través de los cuales la misma humanidad se ennoblece y aparece como divina» (1993; 213). El héroe ético-religioso es el que, inundado de la conciencia de ser nada frente al Ser supremo, sacrifica su vida ofrendándosela. Al manifestar prístinamente el ser como nada del sujeto, la vida religiosa, paradójicamente, termina revelando el aspecto más siniestro de ese Ser mostrándolo como la siempre acechante amenaza de la nada sobre los entes.

Además de la creación objetiva del arte y la sumisión subjetiva de la religión, hay una tercera forma en que, pensándolo, se manifiesta el Ser a la conciencia: la filosofía. La filosofía es objetiva porque muestra el proceso del sujeto creador que, produciéndolos, atraviesa todos los grados de realidad sin estancarse en ninguno, y es subjetiva porque al concebir cómo todo llega a la realidad efectiva muestra también cómo todo el proceso por el cual la totalidad de los entes existe se refiere finalmente al Sujeto primero o Ser (ver 1993; 213). La filosofía muestra la necesidad de lo existente pensándolo como manifestación inmanente del Ser que, como quererse a sí mismo creándose en sus objetivaciones, no se reduce a ninguna de éstas.

La reproducción objetiva del arte, la subjetividad inundada de nada de la religión y la filosofía que piensa la producción efectiva del mundo refiriéndola al Ser por cuyo devenir se sostiene todo, son 
las tres formas de la actividad humana en las cuales se manifiesta el mismo Sujeto supremo que comprende bajo sí a su existencia tanto en la naturaleza como en la historia.

Arte, religión y filosofía son las tres esferas de la actividad humana, en las que sin embargo se manifiesta el espíritu supremo como tal. Él es el genio del arte, el genio de la religión y el genio de la filosofía. Sólo a estas tres esferas se les confiere un carácter divino y, consiguientemente, también una inspiración original (1993; 213).

El artista, el hombre religioso y el filósofo son los distintos creadores que, inspirados por la misma actividad del sujeto primordial, realizan sus actividades manifestando en los resultados de éstas - el arte, la vida religiosa y la obra del pensamiento - a ese mismo Ser como espíritu.

El Ser se manifiesta arrebatando a los inspirados espirituales para que éstos, desde sus obras, señalen y simbolicen la relación de aquél con la conciencia, sin que el contenido que ésta pueda albergar coincida absolutamente con lo que siendo como nada se quiere a sí mismo llegando a ser todo sin que en nada de lo que existe termine por consumarse permanentemente: por su propio huir de la nada, el ser está condenado a devenir eternamente.

En el sistema filosófico, a final de cuentas, el ser mismo es el que, desplegándose en su devenir natural e histórico, termina por acceder a la autoconciencia. «A eso corresponde en concreto la convicción de que lo que conoce en nosotros es lo mismo que lo que es conocido» (1993; 215). Y con el sistema como autoconciencia del Ser que se despliega en la naturaleza y la historia, la naturaleza se descubre como algo autónomo que actúa por sí misma imbuida de un profundo impulso vital, y la historia deja atrás el absurdo de la arbitrariedad caótica y desesperada mostrándose como el ámbito en el que, incesantemente y por lo tanto nunca alcanzado definitivamente, se constituye el sentido de la vida humana (ver 1993; 215).

Schelling, asumiendo el carácter histórico de la constitución moderna del sistema, se ha retrotraído a la profundidad abismal del ser como nada que al quererse como algo se ha constituido en el 
fundamento por el cual existe todo y, mostrando el proceso por el cual este fundamento deviene en su oposición dando lugar a todo lo existente, se ha elevado hasta las cimas de la actividad humana (arte, religión y filosofía) en cuyos productos se manifiesta el mismo ser «como un proceso continuo y necesario» $(1993 ; 214)$. Esta exposición de su propio sistema culmina con un dictamen en el que, detrás de la vanidad, resplandece la honestidad del pensador: pensar contra sí mismo.

Todo el que conozca en su figura genuina y original el sistema últimamente expuesto, se encontrará con él incluso hoy día en una situación particular. Por un lado, le parecerá que este sistema sea falso; por otro lado, sentirá algo que le impida considerarlo al menos como el último sistema verdadero. Lo reconocerá como verdadero dentro de cierto límite, pero no de manera absoluta e incondicionada. Por tanto, para tener un juicio fundado sobre él, habrá que tomar conciencia de estos límites $(1993 ; 214)$.

El propio levantamiento del sistema sustentado en la contradicción entre Ser y entes, sujeto y objeto, infinito y finito, libertad y necesidad, lo confronta con el devenir que, asumido radicalmente, alza la imposibilidad de la clausura en la verdad absoluta.

\section{BIBLIOGRAFÍA}

Schelling, F. W. J. (1993). Lecciones muniquesas para la historia de la filosofía moderna, Luis de Santiago Guervós (trad). Málaga: Edinford. 


\section{RESUMEN}

A partir de Schelling podemos llegar a la conciencia de que no es posible la clausura de la verdad absoluta en los sistemas filosóficos modernos, en cambio, habría que reconocerlos como verdaderos dentro de ciertos límites. Para ello, Schelling sustenta los sistemas en la contradicción entre Ser y entes, i. e. entre sujeto y objeto, infinito y finito, libertad y necesidad. Para Schelling, la contradicción fundamental es que el Sujeto es nada pero se quiere como algo. Como se ve, el sentido del Sujeto cambia en relación a la tradición moderna, premisa importante en Schelling ya que en ella se constituye el nacimiento de la conciencia trágica en la filosofía. Que el sujeto es una actividad contradictoria se muestra al pasar por los siguientes puntos: el principio trágico del ser, la experiencia trágica de la historia y por último, las manifestaciones del Ser.

Palabras clave: Schelling, conciencia trágica; la tragedia del ser; necesidad; libertad; sistema como autoconciencia del Ser.

\section{ABSTRACT}

By Schelling's hand we can realize that the closure of absolute truth in philosophical modern systems is not possible, instead, we should recognize them as true within limits. For this, Schelling defends the systems in the contradiction between Being and entities, i. e. between subject and object, infinity and finite, freedom and necessity. To Schelling, the fundamental contradiction is that the Subject is nothing, but he wants himself as something. As we see, the Subject's sense change in relation to the modern tradition. This is Schelling's important premise being that, in it, the birth of tragic conscience is constituted. The following steps shows that the subject is a contradictory activity: the tragical beginning of Being; the tragical experience of history; and Being's manifestations.

Keywords: Schelling, tragical conscience; the tragedy of Being; necessity; freedom; system as a Being's self-consciousness. 